\title{
Biomarker-Driven Prognostication in Merkel Cell Carcinoma: A Paradigm for Personalized Therapy
}

\author{
Beth A. Helmink, MD, PhD ${ }^{1,3}$, George Ansstas, $\mathrm{MD}^{2,3}$, and Ryan C. Fields, $\mathrm{MD}^{1,3}$ \\ ${ }^{1}$ Section of Surgical Oncology, Department of Surgery, Washington University School of Medicine, St. Louis, MO; \\ ${ }^{2}$ Department of Medical Oncology, Washington University School of Medicine, St. Louis, MO; ${ }^{3}$ Alvin J. Siteman \\ Comprehensive Cancer Center, Washington University School of Medicine, St. Louis, MO
}

While the diagnosis of Merkel cell carcinoma (MCC) is rare, the incidence has almost doubled in the United States since 2000. ${ }^{1}$ Moreover, when MCC has spread beyond the primary tumor, recurrence is high. Until more recent advances in antitumor immunotherapies, treatment options were limited and outcomes were poor. ${ }^{2,3}$

The prevalence of MCC within aged and immunosuppressed, including AIDS and transplant populations, had for many years suggested an infectious etiology. ${ }^{4,5}$ In 2008 , the root cause of MCC in approximately $80 \%$ of patients in the United States was determined to be the oncogenic transformation of cells following a very common childhood viral infection: ${ }^{6}$ Merkel cell polyoma virus $(\mathrm{MCPyV}) .{ }^{4}$ This is the only human polyoma virus known to be associated with malignancy. Oncogenesis requires the clonal integration of $\mathrm{MCPyV}$ and the aberrant production of C-terminally mutated large $\mathrm{T}$ antigen and small $\mathrm{T}$ antigen. ${ }^{4,5}$ The $\mathrm{T}$ antigens are bona fide oncoproteins, promoting cellular transformation in vitro. ${ }^{5}$

The presence of MCPyV itself within a tumor can be identified via immunohistochemistry (IHC) or PCR, thus dividing tumors into virus-positive (VP) and virus-negative (VN) groups. ${ }^{5}$ Antibodies to the oncoproteins are detected in approximately $50 \%$ of VP patients and rarely in the baseline healthy population. ${ }^{7,8}$ This is distinctly different from antibodies to the capsid proteins of the virus, which

(C) Society of Surgical Oncology 2021

First Received: 19 October 2021

Accepted: 25 October 2021;

Published Online: 17 November 2021

R. C. Fields, MD

e-mail: rcfields@wustl.edu can be found in a large fraction of the general population secondary to near ubiquitous exposure. ${ }^{5,6,9}$ Based on these numbers, we would anticipate approximately $40 \%$ of all patients with MCC (50\% of the VP population) to have detectable oncoprotein antibodies (VP seropositive MCC patients).

The ability to detect oncoproteins via serology brings incredible opportunities, especially if these serologies correlate with disease burden and patient outcome. Indeed, this is the case for MCC. Led by Paul Nghiem and his group from the University of Washington, the prognosis of seronegative patients was found to be less favorable with a $42 \%$ higher risk of recurrence for seronegative patients as compared to seropositive patients. ${ }^{8}$ Moreover, viral titers correlate with tumor burden, fall with successful treatment, and rise with recurrence, often before clinical or radiographic detection with a $66 \%$ positive predictive value. ${ }^{7,8}$ These data were collected both in retrospective and prospective fashion and form the basis for the current NCCN guidelines which recommend consideration for MCPyV serologies in all patients with MCC at point of diagnosis and sequentially for ongoing surveillance in $\mathrm{VP}$ patients. $^{7,8,10}$

In this issue of ASO, Arroyave et al. further substantiate these data demonstrating that MCPyV-Ab seropositive patients had improved 2-yr DFS compared with seronegative patients (median 22.2 mo and median not reached, respectively, $p=0.04) .{ }^{11}$ None of the seropositive patients in this cohort experienced a recurrence, whereas 9 of 30 $(30 \%)$ of the seronegative patients eventually recurred. Notably, eight of the seronegative patients never achieved disease clearance; while these patients were excluded for the analyses presented herein, this speaks to the overall poor outcomes in the seronegative patients. Furthermore, 
there was a trend towards improved overall survival in the seropositive patients, but this did not reach statistical significance. This study certainly underscores the importance for collecting this key piece of data in all patients presenting with MCC.

This study does suffer from limitations inherent to all small, single-center, retrospective studies of rare disease with numbers too low for any multivariate analyses. These are not weaknesses in the study per se but do merit a brief discussion. There are some notable baseline differences in disease burden and treatment differences between the seronegative and seropositive groups. ${ }^{11}$ The patients in the seronegative group were older, male, with tumors on the head/neck-all known poor prognostic factors. Although not statistically significant, there also was a greater fraction of immunosuppressed individuals in the seronegative group. There was a higher level of margin-positive excisions in the seronegative population, and $50 \%$ of the seronegative patients did not undergo nodal evaluation due to poor functional status. Moreover, fewer patients in the seronegative group received adjuvant radiation. These latter factors result in not only understaging, but increase the likelihood for detectable locoregional recurrence.

Notably, there was a lower seropositivity rate $(25 \%)$ in the observed population than one would anticipate in allcomers with a diagnosis of MCC. ${ }^{7,8,11}$ The seronegative population should include two distinct populations: (1) the $20 \%$ of all MCC patients not associated with MCPyV whose tumor have tumors that arisen though alternative oncogenic pathways (VN MCC)—for MCC, likely the accumulation of UV-based oncogenic mutations over time; ${ }^{12}$ and (2) the $40 \%$ of total patients who have VP tumors but have, for whatever reason, not mounted an appropriate humoral immune response to these foreign proteins (VP seronegative MCC). This may reflect a skewing of this study's population towards one that is more likely VN than VP being older, male, and with tumors on the head/neck (sun-exposed areas). This distinction is a critical one as it may have important implications in prognosis and potentially even expected treatment outcomes as we discuss.

Interestingly, as the authors point out in the discussion, both VN and VP MCC both respond to immune checkpoint inhibitors (ICIs) at approximately the same relatively high rates with an overall response rate of approximately $50 \%$ to anti-PD-1 blockade and relatively similar breakdown of patients exhibiting partial response, nonresponse, and progression. ${ }^{3,11,13-16}$ This represents one of the highest response rates for solid tumors. ${ }^{3}$ The immunogenicity of these tumors and high responses rates to ICIs for VP and VN tumors, however, is likely driven by different tumor characteristics. ${ }^{3}$ Tumor mutational burden or TMB is a well-known predictor of response to ICIs. VN tumors exhibit a high median TMB of 63.1 mutations per MB ranking among the most highly mutated solid tumors. ${ }^{3,12}$ As is typical of oncogene-driven tumors, however, the tumor TMB of VP MCC is incredibly low at 1.2 mutations per Mb. ${ }^{12}$ However, for VP patients, the antitumor immune response remains strong as it the $\mathrm{T}$ cells are reactive to highly immunogenic foreign viral peptides. ${ }^{3} \mathrm{~T}$ cells recognizing these viral peptides are identified in tumors from patients with MCC. ${ }^{17,18}$

Despite the high response rates to ICIs in many MCC patients, a blanket approach for the use of ICIs for all MCC patients may be short-sighted given the clinical fragility of this patient population. It is likely that the immune responses for individual subgroups (VN, VP seropositive, and VP seronegative) differ somewhat. VP seropositive patients have demonstrated a robust humoral immune response to the viral oncoproteins; these same patients might be predicted to facilitate a strong cellular antitumor immune response as well. ${ }^{3}$ These patients are perhaps most likely to respond to ICIs, and the serologies represent a unique and important tool for surveillance. ${ }^{7,8,11}$ Similar to melanoma, VN tumors with characteristically high TMB may respond to ICIs. ${ }^{3,13-16}$ However, we have no predictive biomarkers as we do in the VP seropositive population and thus no way other than serial imaging to follow the course of disease. The NCCN currently recommends increased radiographic surveillance for these seronegative populations. ${ }^{10}$ However, novel methods for tracking disease burden, including assay for circulating tumor cells (CTCs) and circulating tumor DNA (ctDNA), are being developed and used in the clinical setting and represent an important tool for medical oncologists. ${ }^{19}$ Meanwhile, VP seronegative patients fail to mount an antibody response likely secondary to viral-mediated immunosuppression or inherent host defects in humoral and potentially also cellular immunity. ${ }^{5}$ The virally induced immunosuppression that likely contributed to the lack of a humoral immune response in these individuals may similarly prohibit a response to ICIs (e.g., via MHC Class I downregulation). ${ }^{5,20}$ Host considerations for patients with MCC that might prevent either a humoral response to the viral oncoproteins or a cellular antitumor immune response include aging and chronic immunosuppression, including HIV/AIDS, hematologic malignancies, autoimmune disease, or solid-organ transplantation. For these VP seronegative patients, ICIs may not work well and/or may very well be contraindicated. ${ }^{3,5} \mathrm{We}$ must develop methods to identify those patients most likely to exhibit primary resistance or to develop secondary resistance to ICIs or significant toxicity and work to develop alternative treatment strategies for these individuals. 
As we better select patients most likely to benefit from immunotherapy and least likely to suffer severe side effects, we can move forward more confidently with adjuvant and neoadjuvant treatment strategies for this aggressive tumor. Treating disease at an earlier stage may lead to better outcomes and potentially cure. These studies remain in the clinical trial phase at this time. For adjuvant therapies, the STAMP trial NCT03712605, ADAM trial NCT03271372, ADMEC-O trial NCT02196961, and I-MAT trials are all ongoing. ${ }^{3}$ MCC patients were included in the Phase I/II Checkmate 358 study where 39 patients with PCR-confirmed VP MCC underwent presurgical therapy for advanced Stage IIa to IV MCC. ${ }^{21}$ Approximately $42 \%$ of these VP patients had a pathologic complete response, and none of those individuals had a tumor relapse during follow-up. This is a rather impressive outcome and suggests that we could even potentially further tailor postoperative surgical and nonsurgical therapies in these patients. Unfortunately, no serology data were included, but one might suspect that the rate of pathologic complete response may have been even higher in VP seropositive patients compared with VP seronegative patients.

MCC represents a unique tumor that may be uniquely responsive to immunotherapeutic strategies including ICIs. As in all cases, selecting the patients most likely to exhibit a durable tumor response without incurring significant immune related toxicity is paramount. However, to best treat an individual with MCC, we must have a good understanding of individual's tumor biology/etiology and a basic understanding of the patient's systemic immune competence, which for MCC may include viral serologies.

\section{DECLARATIONS}

DISCLOSURES The authors declare no conflicts of interest.

\section{REFERENCES}

1. Paulson KG, Park SY, Vandeven NA, Lachance K, Thomas H, Chapuis AG, et al. Merkel cell carcinoma: current US incidence and projected increases based on changing demographics. $J$ Am Acad Dermatol. 2018;78(3):457-63. https://doi.org/10.1016/j.jaa d.2017.10.028.

2. Harms KL, Healy MA, Nghiem P, Sober AJ, Johnson TM, Bichakjian CK, et al. Analysis of prognostic factors from 9387 merkel cell carcinoma cases forms the basis for the new 8th edition AJCC staging system. Ann Surg Oncol. 2016;23(11):3564-71. https://doi.org/10.1245/s10434-016-52664.

3. Colunga A, Pulliam T, Nghiem P. Merkel cell carcinoma in the age of immunotherapy: facts and hopes. Clin Cancer Res. 2018;24:2035-43. https://doi.org/10.1158/1078-0432.CCR-170439.

4. Feng H, Shuda M, Chang Y, Moore PS. Clonal integration of a polyomavirus in human Merkel cell carcinoma. Science. 2008;319:1096-100. https://doi.org/10.1126/science.1152586.
5. Pietropaolo V, Prezioso C, Moens U. Merkel cell polyomavirus and Merkel cell carcinoma. Cancers (Basel). 2020. https://doi. org/10.3390/cancers12071774.

6. Martel-Jantin C, Pedergnana V, Nicol JT, Leblond V, Trégouët $\mathrm{DA}$, Tortevoye $\mathrm{P}$, et al. Merkel cell polyomavirus infection occurs during early childhood and is transmitted between siblings. J Clin Virol. 2013;58(1):288-91. https://doi.org/10.1016/j. jcv.2013.06.004.

7. Paulson KG, Carter JJ, Johnson LG, Cahill KW, Iyer JG, Schrama D, et al. Antibodies to merkel cell polyomavirus $T$ antigen oncoproteins reflect tumor burden in merkel cell carcinoma patients. Cancer Res. 2010;70(21):8388-97. https://doi.org/ 10.1158/0008-5472.CAN-10-2128.

8. Paulson KG, Lewis CW, Redman MW, Simonson WT, Lisberg A, Ritter D, et al. Viral oncoprotein antibodies as a marker for recurrence of Merkel cell carcinoma: a prospective validation study. Cancer. 2017;123(8):1464-74. https://doi.org/10.1002/cnc r.30475.

9. Touzé A, Le Bidre E, Laude H, Fleury MJ, Cazal R, Arnold F, et al. High levels of antibodies against merkel cell polyomavirus identify a subset of patients with merkel cell carcinoma with better clinical outcome. J Clin Oncol. 2011;29(12):1612-9. http s://doi.org/10.1200/JCO.2010.31.1704.

10 Bichakjian CK, Olencki T, Aasi SZ, Alam M, Andersen JS, Blitzblau R, et al. Merkel cell carcinoma, version 1.2018, NCCN clinical practice guidelines in oncology. J Natl Compr Canc Netw. 2018;16(6):742-74. https://doi.org/10.6004/jncen.2018.0055.

11. Arroyave AJL, Landry M, McLoughlin JM, Enomoto LM. Merkel cell polyomavirus antibody titer predicts recurrence-free survival. Ann Surg Oncol. (in press)

12. Knepper TC, Montesion M, Russell JS, Sokol ES, Frampton GM, Miller VA, et al. The genomic landscape of Merkel cell carcinoma and clinicogenomic biomarkers of response to immune checkpoint inhibitor therapy. Clin Cancer Res. 2019;25(19):5961-71. https://doi.org/10.1158/1078-0432.CCR-1 8-4159.

13. D’Angelo SP, Russell J, Lebbé C, Chmielowski B, Gambichler T, Grob JJ, et al. Efficacy and safety of first-line avelumab treatment in patients with stage IV metastatic Merkel cell carcinoma: a preplanned interim analysis of a clinical trial. JAMA Oncol. 2018;4(9):e180077. https://doi.org/10.1001/jamaoncol.2018. 0077.

14 Kaufman HL, Russell J, Hamid O, Bhatia S, Terheyden P, D'Angelo SP, et al. Avelumab in patients with chemotherapyrefractory metastatic Merkel cell carcinoma: a multicentre, singlegroup, open-label, phase 2 trial. Lancet Oncol. 2016;17(10):1374-85. https://doi.org/10.1016/S1470-2045(16)30 364-3.

15. Nghiem PT, Bhatia S, Lipson EJ, Kudchadkar RR, Miller NJ, Annamalai L, et al. PD-1 blockade with pembrolizumab in advanced Merkel-cell carcinoma. $N$ Engl $J$ Med. 2016;374(26):2542-52. https://doi.org/10.1056/NEJMoa 1603702.

16. Walocko FM, Scheier BY, Harms PW, Fecher LA, Lao CD. Metastatic Merkel cell carcinoma response to nivolumab. $J$ Immunother Cancer. 2016;4:79. https://doi.org/10.1186/s40425016-0186-1.

17. Afanasiev OK, Yelistratova L, Miller N, Nagase K, Paulson K, Iyer JG, et al. Merkel polyomavirus-specific T cells fluctuate with merkel cell carcinoma burden and express therapeutically targetable PD-1 and Tim-3 exhaustion markers. Clin Cancer Res. 2013;19(19):5351-60. https://doi.org/10.1158/1078-0432.CCR-1 3-0035.

18. Iyer JG, Afanasiev OK, McClurkan C, Paulson K, Nagase K, Jing $\mathrm{L}$, et al. Merkel cell polyomavirus-specific CD8+ and CD4+ $\mathrm{T}$-cell responses identified in Merkel cell carcinomas and blood. 
Clin Cancer Res. 2011;17(21):6671-80. https://doi.org/10.1158/ 1078-0432.CCR-11-1513.

19 Boyer M, Cayrefourcq L, Dereure O, Meunier L, Becquart O, Alix-Panabières C. Clinical relevance of liquid biopsy in melanoma and Merkel cell carcinoma. Cancers (Basel). 2020;12(4):960. https://doi.org/10.3390/cancers12040960.

20 Paulson KG, Voillet V, McAfee MS, Hunter DS, Wagener FD, Perdicchio M, et al. Acquired cancer resistance to combination immunotherapy from transcriptional loss of class I HLA. Nat Commun. 2018;9(1):1-10. https://doi.org/10.1038/s41467-018-06 300-3.
21 Topalian SL, Bhatia S, Amin A, Kudchadkar RR, Sharfman WH, Lebbé $\mathrm{C}$, et al. Neoadjuvant nivolumab for patients with resectable Merkel cell carcinoma in the CheckMate 358 trial. $J$ Clin Oncol. 2020;38(22):2476. https://doi.org/10.1200/JCO.20. 00201.

Publisher's Note Springer Nature remains neutral with regard to jurisdictional claims in published maps and institutional affiliations. 